\title{
COVID-19: Recommendations for Optimum Patient Management and Surgeons' Safety
}

\author{
D BAR $^{\mathrm{a}}$, ABMK ALAM $^{\mathrm{b}}, \mathrm{F}^{\text {RAHMAN }}{ }^{\mathrm{c}}$, AZM MAHFUZUR RAHMAN $^{\mathrm{d}}, \mathrm{N}^{\mathrm{N}}$ SUZANA $^{\mathrm{e}}$
}

Summary:

The novel coronavirus disease 2019 (COVID-19), caused by severe acute respiratory syndrome coronavirus 2 (SARS-CoV2), has evolved rapidly as a global pandemic stretching the healthcare systems worldwide to their limits. This pandemic has resulted in significant changes to surgical practice across the world. Surgeons need to react immediately to this unexpected clinical challenge in a customized and dynamic approach to the selection, screening, planning, and conduct of surgery for their patients.

This current literature will review various international society guidelines to provide a set of recommendations to safely accommodate the exponentially rising cases of SARSCoV-2 infected patients without compromising the care of emergency surgery and oncological patients or jeopardizing the well-being of hospital staff.

Background:

In December 2019, pneumonia of unknown causes was described in the Wuhan, China. ${ }^{1}$ Detailed virological and genomic analyses of infected patients' swab samples subsequently traced these cases to a novel type of corona virus termed SARS-CoV-2, which is responsible for the clinical condition and now the global pandemic referred to as "COVID-19". Due to the rapid global spread of SARS-CoV-2, healthcare systems and their workers worldwide face tremendous challenges, and surgery as a major discipline is by no means an exception. ${ }^{1,2}$

a. Dr. Debashish Bar, Registrar, Department of Surgery, Dhaka Medical College \& Hospital

b. Professor Dr. A.B.M. Khurshid Alam, Professor \& Head, Department of Surgery, Dhaka Medical College \& Hospital

c. Professor Dr. Fatema Rahman, Professor, Department of Gynecology, Dhaka Medical College \& Hospital

d. Dr. A.Z.M. Mahfuzur Rahman, Associate professor, Department of Surgery, Dhaka Medical College \& Hospital

e. Dr. Nawreen Suzana, FCPS part-II trainee, Department of Surgery, Dhaka Medical College \& Hospital

Address of Correspondence: Dr. Debashish Bar, Registrar, Department of Surgery, Dhaka Medical College Hospital, Dhaka, Bangladesh.

Mobile: 01914123469, E-mail: debashishbar01@gmail.com
The recommendations include guidance for selection and screening of patients during different phases of COVID-19 spread throughout the country. Personal protective equipment requirements for safety measures are also reviewed for each phase as minimum requirements. We also provide recommendations on the patient prioritization for surgery, selection of surgical techniques to reduce transmission and exposure risk, operating room set up and conduct of general surgery.

During the COVID-19 pandemic, every surgical unit needs to follow clear guidelines to ensure both patient and staff safety. These current guidelines may assist to provide guidance to units in developing their own protocols.

Keywords: SARS-CoV-2. COVID-19. Guidelines. Recommendations

(J Bangladesh Coll Phys Surg 2020; 38: 116-121) DOI: https://doi.org/10.3329/jbcps.v38i0.47343

Preparations for the developing crisis have initially centred on the provision of intensive care capacities to ventilate seriously ill patients and the maintenance of an adequate supply of protective equipment for medical personnel. As a result, hospital authorities have demanded postponement or even thorough cancellation of all elective operations. ${ }^{3}$ So far, these measures have failed to take into account SARS-CoV-2 positive patients whose main symptoms of disease are not specific to COVID-19 (e.g. respiratory symptoms, fever) but with surgical disease (e.g. acute surgical emergencies, malignancies). These patients must therefore be treated primarily surgically. ${ }^{4}$

Surgery during the period of COVID-19 pandemic faces the challenges in everyday clinical practice like prioritization of surgical interventions, establishment of SARS-CoV-2 and non-SARS-CoV-2 emergency rooms, establishment of a SARS-CoV-2 surgical non-intensive care ward, establishment of a surgical SARS-CoV-2 operating area and necessary precautions when using certain surgical techniques. ${ }^{5}$

Healthcare workers are on the front lines of caring for patients with COVID-19 and have very high risk of exposure to the virus. Shortages of protective equipment and knowledge regarding COVID-19 are causing 
infections in healthcare workers. This strongly suggests that adequate knowledge about disease transmission and use of protective gear and infection control protocols are essential to prevent spread of infection among healthcare workers. Although surgeons are not frontline health workers, there is evidence that several series of infections emerged from operating theaters in China. ${ }^{1}$

The purpose of this article is to provide recommendations for the optimum management of surgical patients during this pandemic. It will also provide guidelines to surgeons and other healthcare workers involved in perioperative care to improve staff and patient safety during this catastrophe. These recommendations are derived from some of the hospitals' practices and various national guidelines for health professionals.

\section{Outpatient management}

Most hospitals need to cancel or reduce nonurgent outpatient visits as part of their COVID-19 containment strategy. Surgeons should prioritize urgent or emergency visits and procedures. Elective and nonurgent admissions need to be rescheduled. ${ }^{6}$ Patients who will face life threatening consequences due to delayed treatment should be prioritized for outpatient visit, telephone or virtual consultation by a member of the surgical team. It is also advisable to set up a separate triage area or fever clinic to screen for respiratory symptoms in any surgical patient. ${ }^{7}$

Patient with respiratory symptoms and flu-like symptoms need to visit the fever clinic in advance. Suspected or confirmed cases of COVID-19 should be assigned private rooms and should be provided surgical face masks without exhalation valve. ${ }^{8}$ Safe distancing in between patients, patient and hospital staffs and between hospital staffs is essential within clinics and hospitals. Doctors and patients should stay at least $6 \mathrm{ft}$ apart except during examinations; it may be necessary to waive all but the most essential elements of the physical exam to minimize risk of transmission. ${ }^{7}$

In addition to collecting routine disease-related information, staff should take a detailed epidemiologic history including the recent travel history of patient and their family members and contact history with people from endemic regions. The staff should also screen for common symptoms of COVID-19 like fever, cough, dyspnea and diarrhoea. According to the Chinese national guidelines, it is recommended to do blood test for COVID-19 and chest CT scan as routine examinations for patients requiring admission. ${ }^{9}$ Even in other countries, it is desirable to advise RT-PCR for COVID-19 among all patients being admitted to hospital for surgery.

\section{Establishment of SARS-CoV-2 and non-SARS-CoV-2 emergency rooms}

To avoid the transmission of SARS-CoV-2 between patients, from patients to healthcare workers and physicians, and to conserve personal protective equipment (PPE), emergency rooms (ER) should be divided into infectious (SARS-CoV-2 suspected and confirmed patients; COVID area) and noninfectious parts (non-COVID area). Checkpoints at the entrance of the ER are set up to assess patients for symptoms of COVID-19, provide them with surgical masks before entering the hospital and guide them to COVID or nonCOVID areas. The assessment of patients regarding SARS-CoV-2/COVID-19 includes a short questionnaire about typical symptoms for COVID-19, potential contact to SARS-CoV-2 positive tested persons, residence in an area with evidenced outbreak of COVID-19 and wireless body temperature monitoring. Every patient in the COVID area must be swabbed for SARS-CoV-2 PCR testing. Depending on the test result and further medical/ surgical treatment, the patient will then be transferred to an operation room, intensive care unit or hospital ward, which are also separated into SARS-CoV2 or nonSARS-CoV-2 areas. If it is medically indicated, patients can be also discharged from the hospital in accordance with local restrictions. ${ }^{10}$

\section{Hierarchical prevention and control of inpatients}

Surgical patients need to be classified into three risk categories for COVID-19: confirmed and suspected patients, high-risk patients, and low-risk patients. They are defined as follows:

1. Confirmed and suspected patients: COVID-19 was confirmed by real-time reverse transcriptase (RT)-PCR test of throat swab or serological (IgM and $\operatorname{IgG}$ ) test results was positive. The definition of suspected cases falls into two categories. The first category will have contact history and meet any two of the clinical manifestations (fever and respiratory symptoms) with the typical findings of COVID-19 in the chest CT scan 
or the typical blood count for COVID-19 (the total number of white blood cells in the early stage of the disease is normal or decreased, and the lymphocyte count is reduced). The second category is without a clear contact history and shows three of the clinical manifestations (fever and/or respiratory symptoms, typical findings in the chest CT, the typical blood count for COVID-19).

2. High-risk patients: Patients who had traveled to highrisk areas or contacted with confirmed or suspected COVID-19 patients who have developed fever or symptoms of acute respiratory illness within 14 days.

3. Low-risk patients: Patients with no history of close contact with confirmed and suspected COVID-19 patients and with no fever or respiratory symptoms and without CT manifestations of COVID-19 within 14 days. ${ }^{11}$

The risk level of all surgical patients should be evaluated before or immediately after admission to hospital and the treating team should evaluate the patient's risk level daily. High-risk, confirmed, and suspected patients must be kept in a single isolation room, and all the necessary disinfection and isolation measures should be implemented. Emergency isolation wards also need to be set up in all hospitals to treat newly admitted highrisk, confirmed, and suspected patients. ${ }^{11,12}$ Elective surgeries for confirmed and suspected patients should be rescheduled, as they have a higher risk of severe events following surgery and should be assessed daily for progression of the disease. For high-risk patients, surgeons should consider both medical and logistical needs but in low-risk patients, elective surgeries should not be abandoned unless there are constrained health resources. For cancer patients who have to delay surgery, alternative treatment approaches can be considered, such as neoadjuvant chemotherapy or additional chemotherapy. ${ }^{13}$

\section{Protocols for emergency surgery}

During this endemic of COVID-19, need for emergency surgery should be considered as a priority for admission. ${ }^{14}$ Surgeons should schedule surgery based on the severity of threat to the patient's life and health. After admission, different management protocols will be applied based on the COVID-19 risk level of patients. Confirmed and suspected patients requiring emergency surgery will be admitted in the isolation ward. Suspected patients need to complete COVID-19 blood test and chest CT scan before admission; pharyngeal swab RT-
PCR sampling should be completed before surgery. For these patients surgeons need to report to the operating theater before surgery, then the patient is transferred to a negative pressure operating theater via a designated path and after the operation, the patient is transferred to the isolation area again. High-risk patients are returned to the original isolation ward and low-risk patients are transferred to the original ward according to the original transfer route after operation. All surgery should be performed in a quick and efficient manner. ${ }^{15}$

Surgeons should also be prepared to encounter COVID19 in three key areas and should respond accordingly. First, patients admitted for treatment of COVID-19 may develop additional problems that require surgical intervention; non-operative treatment options should be considered carefully for these patients, as the resources are limited and survival after major surgery is unlikely. Second, patients admitted with acute surgical pathologies, may have concurrent COVID-19 infection; need for emergency surgery in these patients should be considered as a priority. Third, after surgery patients may develop respiratory symptoms or fever, indicating nosocomial COVID-19 infection; these patients should be isolated early and individuals at risk of exposure are tested rapidly. ${ }^{16}$

\section{Protocols for elective surgery}

American College of Surgeons (ACS) advised to postpone no urgent elective surgeries during the beginning of the pandemic of COVID-19. They have classified surgeries into various tiers according to the urgency. Up to Tier $2 b$ (most elective surgeries: asymptomatic surgical patients, patients with mild symptoms, low risk cancer patients), they are advising to postpone the surgery. For Tier $3 \mathrm{a}$ and $3 \mathrm{~b}$ (most cancer surgeries and highly symptomatic surgical patients), ACS is not advising postponement at this moment but it may change. ${ }^{17}$

According to guidelines from the Indian Council of Medical Research, all high-risk patients undergoing elective surgery should undergo PCR test for COVID19 before surgery. If the patient's RT PCR test is twice negative, surgeons can proceed with regular surgical protocols. If the patient's RT PCR test is positive, then the patient needs to be transferred to the isolation ward to complete the preoperative preparation and elective surgery should be deferred until the patient recovers. ${ }^{18}$ 


\section{Cancer care}

Surgical cancer care poses unique dilemmas, because delayed diagnosis and definitive treatment could worsen oncological outcomes and will cause distress for patients and their families. The duration of pandemic-related disruption is unpredictable so, although postponing cancer care increases immediate hospital capacity, treatment delays may be prolonged. ${ }^{12}$ From the recommendations of the Society of Surgical Oncology, decisions must be made on an individual case basis considering the biology of each cancer, alternative treatment options, and waiting time for rescheduled surgery. ${ }^{19}$ Radiological and endoscopic investigations for patients triaged by telephone with prioritized highrisk symptoms should continue for as long as possible, and elective cancer surgery should be offered to as many patients as possible. Patients at high risk of COVID-19 complications, such as the frail and elderly, can be offered neoadjuvant treatments while definitive surgical management is delayed. ${ }^{15}$

\section{Management during surgery (suspected emergency and test positive elective cases)}

During any surgical intervention or any invasive procedures irrespective of aerosol generating or not, all the theatre staffs have the highest possibility of being infected. ${ }^{13}$ So certain recommendations are made for the safety of the theatre staffs: 1 . The operating area should have anterooms with a negative pressure system, 2. Materials or objects not essential for the operation (e.g. pens, telephones, keys) should remain outside the operating area and the materials required for the operation should all be disinfected and immediately disposed of after use, 3. Have minimum number of personnel in the operating room, no visitors or observers allowed in the OR, 4. Entering and leaving the operating room should be kept to an absolute minimum, 5. The patient should not be moved to a post-anaesthesia recovery room rather than transferred directly to the SARSCoV- 2 surgical ward or intensive care unit, 6. Surgeons and personnel not needed for intubation should remain outside the operating room until anesthesia induction and intubation are completed, 7. All surgery should be performed in a quick and efficient manner, 8. Dual consultant operating and reduction in training procedures should be considered to decrease operating times if operating resources are severely limited. ${ }^{20}$
Though it remains unclear if the virus is able to spread in the peritoneal cavity or other bodily fluids, all objects that come in contact with patients including blood, secretions, and excreta liberated during surgery should be considered as potentially contaminated. ${ }^{19}$ In particular, medical staff in operating theater should avoid exposure to aerosols generated during the use of electrosurgical equipment. Though it is not proven that coronavirus can be transmitted via surgical smoke, it may be wise to take precaution until we have strong evidence it does not. ${ }^{20}$ To reduce the hazards, surgical smoke should be minimized by suction device, and electrosurgical equipment should be used at the lowest effective power. It is also advised that in suspected cases, laparoscopy should be avoided as high-pressure trocar leaks from pneumoperitoneum may enhance the risk of exposure to aerosol to operating theater staff. Surgeons and nurses should also be careful to avoid injuries like stab wounds and needle stick injuries. ${ }^{21}$

All the operations of suspected and test positive cases should be performed in the negative pressure operation theatre to minimize the risk of exposure to aerosol to theater staff. ${ }^{19}$ After completion of surgery laminar air flow of the operating room should be stopped. Peroxyacetic acid air is used for fumigation of the theatre. The operating theater should be cleaned and disinfected, high-efficiency filter should be changed. Cleansing of all hard surfaces in the operating theater should be done using detergent and water followed by use of $1000 \mathrm{ppm}$ bleach solution. The disinfection time should be at least $30 \mathrm{~min}$ and the operating theater should be closed for at least $2 \mathrm{~h}$. The next operation should be performed after laminar flow and ventilation being turned on. ${ }^{22}$

\section{Postoperative management}

For suspected or confirmed patients, adequate oxygen therapy and nebulization should be ensured in the postoperative ward as well as surgeons should pay attention to nutritional supplement and organ support treatment for all postoperative patients. ${ }^{23}$ As these patients have a greater risk of complications such as deep vein thrombosis (DVT) and secondary pulmonary infections, early prophylactic measures should be taken. For high-risk patients who develop cough with fever after surgery, a chest CT scan and RT-PCR for COVID19 test should be advised. ${ }^{22}$ 
For confirmed COVID-19 patients, once the temperature returns to normal for more than 3 days, the respiratory symptoms are significantly relieved, and the inflammation is clearly absorbed, the isolation can be released when the RT PCR test is negative on two consecutive occasions at least $24 \mathrm{~h}$ interval. ${ }^{23}$ These patients then may be transferred to the general ward for treatment or discharge.

\section{Safety Measures for Healthcare Workers}

From the experiences of some hospitals in Wuhan, surgeons are at high risk of infection. Healthcare workers must take preventive measures for personal safety in strict accordance with the epidemic assessment level. ${ }^{13}$ Due to the limited supply of personal protective equipment (PPE) in many centers, their use should be determined by the risk level of each patient. In times of extreme shortages, alternatives to PPEs should be considered.

(1) When entering the ward of low-risk or high-risk patients for daily activities and rounds, primary protection should be needed: disposable surgical cap, surgical mask, work uniform, disposable latex gloves or/and disposable isolation clothing.

(2) When carrying out routine activities and rounds with confirmed and suspected patient wards, secondary protection should be needed: disposable surgical cap, N95 mask, work uniform, disposable latex gloves, disposable medical protective uniform and goggles.

(3) For special procedures such as collecting airway samples, tracheal intubation, airway care, and sputum suction and in the aerosol generating environment, tertiary protection equipments should be used: disposable surgical cap, N95 mask, work uniform, disposable latex gloves, disposable medical protective uniform, full-face respiratory protective devices or powered air-purifying respirator. ${ }^{24}$

Healthcare workers shall strictly follow the procedures for donning and doffing of personal protective gear, and it is forbidden to wear PPEs when one leaves the contaminated areas. Appropriate disposal of PPE should be ensured. Sanitation and disinfection of the hospital environment need to be implemented according to the regionalized zoning management system and patient epidemic classification. ${ }^{22}$

Conclusion
The dynamic prioritization of SARS-CoV-2 infected and surgical patient groups is the key to preserving life while maintaining high surgical standards. With the increasing number of COVID-19 cases worldwide, there is an ongoing need for infection prevention and control. Strictly segregating patient groups in emergency rooms, nonintensive care wards and operating areas prevents viral spread while adequately training and carefully selecting hospital staff allow them to confidently and successfully undertake their respective clinical duties. These principles are essential for both patient care and staff safety. The general principles outlined in this report will need to be considered in light of the local context, available resources, and the current activity of COVID-19.

\section{Conflict of Interests}

The authors declare that they have no conflict of interest.

\section{References}

1. Huang C, Wang Y, Li X, Ren L, Zhao J et al. Clinical features of patients infected with 2019 novel coronavirus in Wuhan, China. Lancet 2020; 395(10223):497-506.

2. World Health Organization. Coronavirus disease 2019 (COVID-19) Situation Report. https://www.who.int/docs/ default-source/coronaviruse/situation-reports/20200318sitrep-58-covid-19.pdf?sfvrsn=20876712_2

3. Hellewell J, Abbott S, Gimma A. Feasibility of controlling COVID-19 outbreaks by isolation of cases and contacts. Lancet 2020; Glob Health. https://doi.org/10.1016/S2214109X(20)30074

4. Huh S. How to train the health personnel for protecting themselves from novel coronavirus (COVID-19) infection during their patient or suspected case care. J Educ Eval Health Prof 2020; 17:10.

5. Zhonghua Liu, Xing Bing, Xue Za Zhi. The epidemiological characteristics of an outbreak of 2019 novel coronavirus diseases (COVID-19) in China. Novel Coronavirus Pneumonia Emergency Response Epidemiology Team 2020; 41(2):145-151

6. Livingston E, Bucher K. Coronavirus disease 2019 (COVID19) in Italy. JAMA 2020. https://doi.org/10.1001/ jama.2020.4344

7. National Health Commission of the People's Republic of China. Joint prevention and control mechanism of the state council. (20 Mar 2020) http://www.gov.cn/xinwen/ gwylflkjz63/wzslqt.htm,

8. Centers for Disease Control and Prevention. Resources for clinics and healthcare facilities. (16 March 2020). https:// www.cdc.gov/coronavirus/2019-ncov/healthcare-facilities/ index.html 
9. National Clinical Programme in Surgery: Information for surgeons regarding OPD triage during COVID-19 epidemic. (19 March 2020) https://msurgery.ie/wp-content/uploads/ 2020/03/v3-NCPS-guidance-tosurgeons-for-OPD-triageduring-COVID-19.pdf

10. Centers for Disease Control and Prevention: Interim guidance for healthcare facilities: preparing for community transmission of COVID-19 in the United States. (19 February 2020). https://www.cdc.gov/coronavirus/2019-ncov/ healthcare-facilities/guidance-hcf.html

11. Liang W, Guan W, Chen R, Wang W, Li J et al. Cancer patients in SARS-CoV-2 infection: a nationwide analysis in China. Lancet Oncol 2020; 21(3):335-337.

12. Walker JL, Piedmonte MR, Spirtos NM, Eisenkop SM, Schlaerth JB. Laparoscopy compared with laparotomy for comprehensive surgical staging of uterine cancer: Gynecologic Oncology Group Study LAP2. J Clin Oncol 2020; 27(32):5331-5336.

13. Hensman C, Baty D, Willis RG, Cuschieri A. Chemical composition of smoke produced by high-frequency electrosurgery in a closed gaseous environment. Surg Endosc 1998; 12:1017-1019.

14. Johnson GK, Robinson WS. Human immunodeficiency virus1 (HIV-1) in the vapors of surgical power instruments. J Med Virol 1991; 33:47-5015.

15. Mellor G, Hutchinson M. Is it time for a more systematic approach to the hazards of surgical smoke? Reconsidering the evidence. Workplace Health Saf 2013; 61(6):265-270.
16. American College of Surgeons. COVID-19: guidance for triage of non-emergent surgical procedures. (24 March 2020). https://www.facs.org/about-acs/covid-19/ information-for-surgeons/triage

17. American College of Surgeons (2020) COVID 19: elective case triage guidelines for surgical care. (Assessed $25 \mathrm{Apr}$ 2020). https ://www.facs.org/covid -19/clini cal-guida nce/ elect ive-case.

18. Robert-Koch-Institut. (2020). https://www.rki.de/DE/ Content/ I n f A Z / N / N e u a r tige s_C oronavir u s/Getre n n t e_Patientenversorgung.html.

19. Ti LK, Ang LS, Foong TW, Ng BSW. What we do when a COVID-19 patient needs an operation: operating room preparation and guidance. Can J Anaesth 2020.

20. Royal College of Surgeons (2020) Updated general surgery guidance on COVID-19 (15 Apr 2020). https ://www.rcsen g.ac.uk/coron aviru s/joint-guida nce-for-surge ons-v2/.

21. Morrison JE Jr, Jacobs VR. Replacement of expensive, disposable instruments with old-fashioned surgical techniques for improved cost-effectiveness in laparoscopic hysterectomy. JSLS 2020; 8(2):201-206 22.

23. Technology Committee of the European Association for Endoscopic Surgery, A low cost, safe and effective method for smoke evacuation in laparoscopic surgery for suspected coronavirus patients. (2020). https ://doi.org/10.1097/ SLA.00000 00000003965

24. American College of Surgeons COVID-19: considerations for optimum surgeon protection before, during, and after operation. (2020). https ://www.facs.org/covid -25 